\title{
Study on the Optoelectronic Properties of UV Luminescent Polymer: ZnO Nanoparticles Dispersed PANI
}

\author{
J. Kayathri, N. RaniMeiyammai, S. Rani, K. P. Bhuvana, K. Palanivelu, and S. K. Nayak \\ Central Institute of Plastics Engineering \& Technology, Chennai 600 032, India \\ Correspondence should be addressed to K. P. Bhuvana; kpusha27@gmail.com
}

Received 16 November 2012; Revised 22 January 2013; Accepted 25 January 2013

Academic Editor: Amit Bandyopadhyay

Copyright ( 2013 J. Kayathri et al. This is an open access article distributed under the Creative Commons Attribution License, which permits unrestricted use, distribution, and reproduction in any medium, provided the original work is properly cited.

\begin{abstract}
The present work is focused on the synthesis and characterization of a new polymer thin film material suitable for emissive layer in polymer light-emitting diodes. Thin films of polyaniline and $\mathrm{ZnO}$ nanoparticles dispersed polyaniline have been prepared and investigated for the suitability of emissive layer in polymer light emitting diodes. $\mathrm{ZnO}$ nanoparticles have been synthesized using wet chemical method and characterized using powder X-ray diffraction. Diffraction pattern reveals that the ZnO nanoparticles are grown in hexagonal wurtzite structure with the preferential orientation of (001) plane. Polyaniline was synthesized using potassium persulphate (initiator) at room temperature. $\mathrm{ZnO}$ nanoparticles are then dispersed in different doping level (5\%, 10\%, 15\% 20\% and $50 \%)$ with polyaniline. Thin films of $\mathrm{ZnO}$ dispersed polyaniline (PANI/ZnO) were prepared and characterized using scanning electron microscopy in order to investigate the surface morphology of the film. The optical characteristics and the suitability of film as emissive layer were analyzed using UV-visible absorption and fluorescence spectroscopy.
\end{abstract}

\section{Introduction}

After the discovery of conductivity in polymers and in certain organic molecules, remarkable progress has been made in synthesizing organic materials and in developing them for use in electronic and optical devices $[1,2]$. Currently, polymer-based organic light-emitting diodes (LEDs) [3], photovoltaic cells [4], and field effect transistors [5] are being pushed towards commercialization. So far, a wide range of conjugated polymer systems has been developed, such as poly (1,4-phenylenevinylene) (PPV) [6, 7], poly(p-phenylene) (PPP) [8], polyfluorene (PF) [9], and polythiophene (PT) [10] and their derivatives. Polymer-based optoelectronic devices have gained importance due to the cost-effective nature of polymers. Polymer light-emitting diodes (PLEDs) were first demonstrated by Richard Friend and coworkers on PPV that emitted 3 primary colors. Among the conducting polymers, PANI has been of particular interest because of its cheap monomer, simple synthesis technology, unique electrochemical properties, high conductivity, and environmental stability. PANI differs from most other conducting polymers such as PPV and PT because it possesses readily accessible oxidation states. Zinc oxide has received much attention in recent years due to its diverse properties. It is a direct wide gap semiconductor, and it is a prominent inorganic material for optoelectronic devices. The interest of the researchers has increased rapidly during the last decade in the application of inorganic-organic hybrid polymers for electronic and optoelectronic devices. A new finding of a nanocrystal $\mathrm{ZnO}$ retarded gelation of low-density polyethylene (LDPE) has also been reported [11]. It has been reported that nanocrystals of inorganic materials exhibit many properties which are unexpected from the conventional point of view. PLEDs by depositing a p-type polymer, poly(3,4-ethylenedioxythiophene): poly(styrene sulfonate)(PEDOT:PSS) on $\mathrm{n}$-type $\mathrm{ZnO}$ nanowires grown by electrodeposition on transparent conducting glass (ITO) substrates have also been fabricated and reported [12]. Incorporation of few wt $\%$ of $\mathrm{ZnO}$ nanoparticles in polymer matrix has enhanced its emissivity. The present investigation is focused on the hybridization of PANI with $\mathrm{ZnO}$ and analyzing its optical properties as an emissive layer of LED. 


\section{Experimental}

Thin films of PANI and PANI/ZnO have been synthesized by solution polymerization technique with different compositions of $\mathrm{ZnO}(5,10,15,20$ and $50 \mathrm{wt} \%) .0 .1 \mathrm{M}$ of zinc acetate was added in starch solution. Starch solution is prepared by adding $2.5 \mathrm{~g}$ of starch in $250 \mathrm{~mL}$ of distilled water. After complete dissolution of zinc acetate under constant stirring, ammonia $(0.2 \mathrm{M})$ is added dropwise to the aforemention solution. The obtained solution is then filtered and dried in oven at $80^{\circ} \mathrm{C}$ to get $\mathrm{ZnO}$ nanoparticles. PANI was synthesized by solution polymerization technique. Prescribed amount of aniline $(5.58 \mathrm{~g})$ is dissolved in $1 \mathrm{~N}$ hydrochloric acid under constant stirring for 4 hours. Calculated amount of potassium persulphate, $\mathrm{K}_{2} \mathrm{~S}_{2} \mathrm{O}_{8}(1.62 \mathrm{~g})$, in $10 \mathrm{~mL}$ of distilled water is added to aniline in $\mathrm{HCl}$ solution and constantly stirred for 24 hours [13]. The polymerization solution results in the formation of dark green precipitate. The precipitate is then filtered and dried in oven at $110^{\circ} \mathrm{C}$ to get PANI powder. $\mathrm{ZnO}$ nanoparticles at different composition were dispersed into PANI by adding $\mathrm{ZnO}$ nanoparticles to the aqueous solution of aniline in $\mathrm{HCl}$ after adding the initiator while the solution is in constant stirring. PANI and PANI/ZnO composites are then powdered in mortar and preserved in a desiccator.

Thin films of PANI and PANI/ZnO were prepared by solution method. The PANI/ZnO powdered composite was mixed with polyvinyl alcohol (PVA) solution (1 gm of PVA in $20 \mathrm{~mL}$ of distilled water). The PVA solution was used as a binding agent. The obtained solution is coated on a transparent glass substrate by spinning method on glass substrate. The solution was allowed to fall dropwise, and the substrate was placed in a rotating disc of the abrasion tester. The disc was set to rotate with the speed of $100 \mathrm{rpm}$ for $2 \mathrm{hrs}$. The solution was deposited on the glass substrate. The film was kept in the oven for 2 hrs by maintaining the temperature of $150^{\circ} \mathrm{C}$. The thickness of the films was measured using digital foil thickness gauge (Make: Precision, Model: 500-FD, Accuracy: $1.0 \mu \mathrm{m}$ ) and found to be around 310-350 $\mu \mathrm{m}$.

\section{Results and Discussion}

3.1. Density. The density of the nanocomposites was determined using the formula $D_{C}=v D_{\mathrm{ZnO}}+(1-v) D_{\mathrm{PANI}}$. The density of $\mathrm{ZnO}$ nanoparticles and PANI was determined as $5.59 \mathrm{~g} / \mathrm{cc}$. and $1.32 \mathrm{~g} / \mathrm{cc}$., respectively. The density of PANI/ZnO nanocomposites with different composition of $\mathrm{ZnO}$ was determined using the previously formula, and the values are tabulated in Table 1.

\subsection{Structural Characteristics}

3.2.1. X-Ray Diffraction Studies. The structural properties of as-synthesized $\mathrm{ZnO}$ nanoparticles and PANI were investigated using X-ray diffraction (XRD). The XRD pattern was recorded in Shimadzu diffractometer, using the $\mathrm{Cu}$ $K_{\alpha}$ radiation, $\lambda=1.5418 \AA$ with $40 \mathrm{kV}$ and $20 \mathrm{~mA}$, at $0.04^{\circ}$ scan rate. The measurements were made at room temperature with the diffraction angle $(2 \theta)$ ranging from
TABLE 1: Density of PANI/ZnO nanocomposites.

\begin{tabular}{lc}
\hline Concentration of ZnO in PANI/ZnO nanocomposites & $\begin{array}{c}\text { Density } \\
\text { (g./cc.) }\end{array}$ \\
\hline $0 \%$ & 1.320 \\
$5 \%$ & 1.534 \\
$10 \%$ & 1.747 \\
$15 \%$ & 1.961 \\
$20 \%$ & 2.174 \\
$50 \%$ & 3.455 \\
\hline
\end{tabular}

10 to 70 degrees. XRD pattern of $\mathrm{ZnO}$ nanoparticles and PANI is depicted in Figure 1. The XRD pattern of PANI shows the amorphous nature of PANI; however the XRD pattern of $\mathrm{ZnO}$ nanoparticles reveals that they are grown in hexagonal wurtzite structure with the preferential orientation of (100) plane. The diffraction peaks do not imitate the high crystallinity; hence the size of the particles must be smaller. Inset of Figure 1 shows the Williamson-Hall plot which was to calculate the particle size and the strain induced due to the reduction of size of the particle.

From Williamson-Hall plot it is observed that the particle size $(D)$ and the strain $(\varepsilon)$ were calculated as $4.496 \mathrm{~nm}$ and -0.0685 , respectively. The negative sign represents that the strain induced is compressive. Moreover, the lattice constants " $a$ " and " $c$ " are determined using the following formulae:

$$
a=\frac{\lambda}{\sqrt{3} \sin \theta}, \quad c=\frac{\lambda}{\sin \theta} .
$$

The " $a$ " axis lattice constant and " $c$ " axis lattice constant are determined to be $a=3.1606 \AA$ and $c=5.4743 \AA$. The bulk $\mathrm{ZnO}$ has the lattice constant of $a=3.25 \AA$ and $c=5.206 \AA$. Along " $a$ " axis the particle undergoes the compressive strain, and hence the lattice constant is reduced from that of the bulk. However, the $c$ axis lattice constant is increased. The reason is that, when there is a compressive strain on one direction, there will be a tensile strain on the opposite direction. Thus the lattice constant is increased along $c$ axis. The average particle size using Debye-Scherer formula is around $3.93 \mathrm{~nm}$.

3.2.2. SEM Analysis. The distribution of $\mathrm{ZnO}$ particles was examined using scanning electron microscopy. The SEM images of PANI/ZnO films coated on glass substrate were depicted in Figure 2. The SEM images have been recorded using Carl Zeiss scanning electron microscope at the magnification of $8.00 \mathrm{Kx}$. The SEM images clearly reveal the uniform dispersion of $\mathrm{ZnO}$ nanoparticles in PANI matrix. Further it is observed from SEM image that the $\mathrm{ZnO}$ particles exist in the form of hexagonal rod. Moreover, the distribution increases with the increase of $\mathrm{ZnO}$ concentration into the PANI matrix. PANI with $20 \%$ of $\mathrm{ZnO}$ particles shows the higher degree of uniform distribution. However, at higher concentrations of $\mathrm{ZnO}(50 \%)$, the particles are clustered together due to the stronger intermolecular forces between them. 

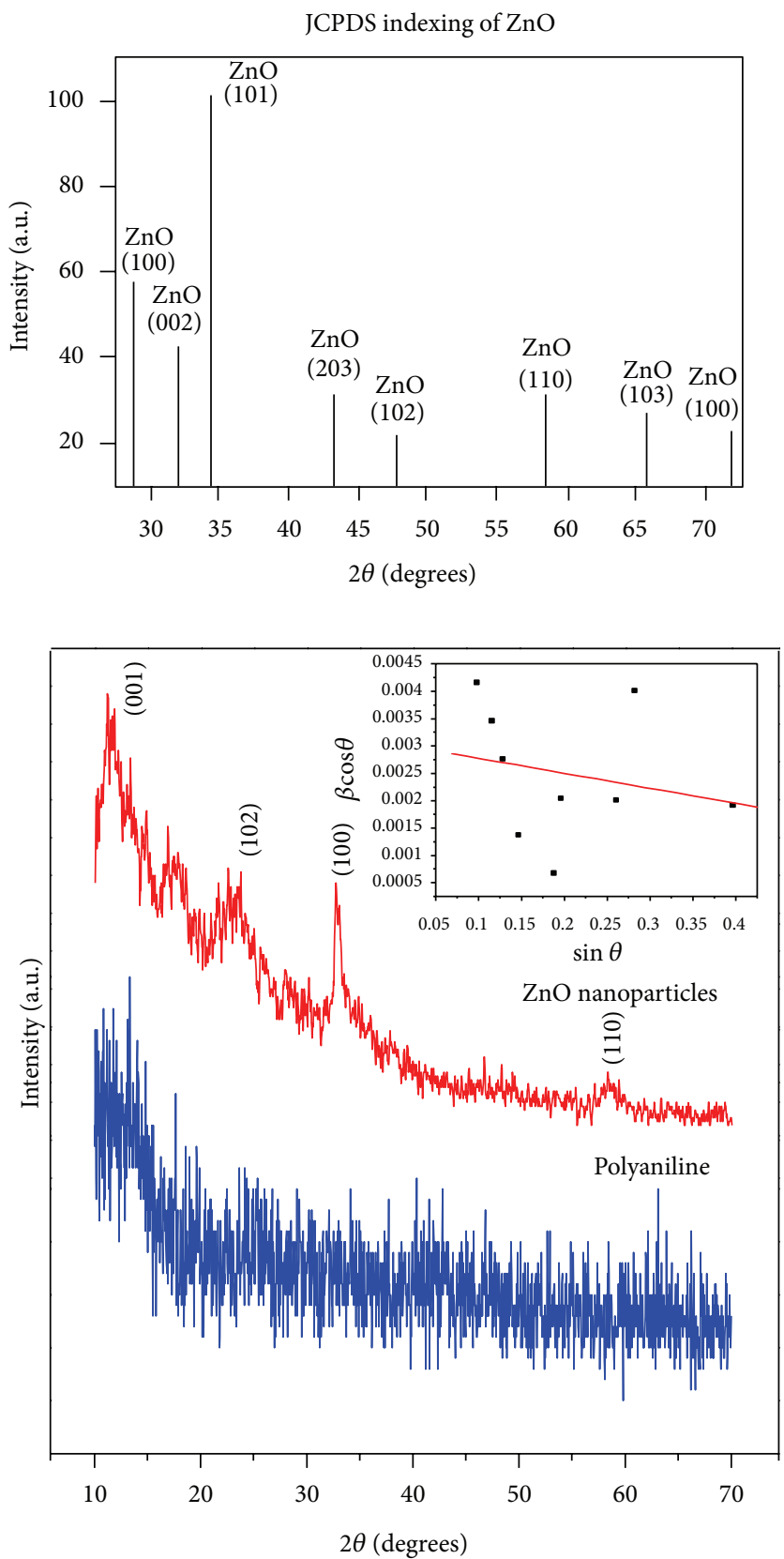

FIGURE 1: XRD pattern of PANI and $\mathrm{ZnO}$ nanoparticles. Inset: W-H plot for $\mathrm{ZnO}$ nanoparticles.

\subsection{Optical Characteristics}

3.3.1. Optical Transmittance. Optical transmittance data was observed using Clarity meter (Make: Gardner Laboratory, Inc., USA) which emits the light radiation of wavelength $860 \mathrm{~nm}$. Figure 3 shows the variation of optical transmittance as a function of concentration of $\mathrm{ZnO}$ nanoparticles. It is observed that the incorporation of $\mathrm{ZnO}$ nanoparticles into the PANI matrix increases the transparency of the PANI film.

3.3.2. Determination of Optical Band Gap. UV-visible absorption spectra were observed using CARY 5E UV-VISNIR spectrophotometer at room temperature within the wavelength ranges from $300 \mathrm{~nm}$ to $1100 \mathrm{~nm}$ in steps of $1 \mathrm{~nm}$. The optical band gap as a function of $\mathrm{ZnO}$ nanoparticles concentration has been extracted from UV-Visible spectrum. The transmittance data from the UV spectrum have been used for the further determination of optical parameters like band gap and refractive index.

To determine the optical band gap of the composites, Tauc's plot for pure PANI and PANI/ZnO composites was plotted and depicted in Figure 4. From the graph, it is observed that the optical band gap decreases with respect to the concentration of $\mathrm{ZnO}$. Since $\mathrm{ZnO}$ is a direct band gap semiconductor, Tauc's plot is plotted between $(\alpha h v)$ and $h v$. The band gap is determined by extrapolating the linear region 


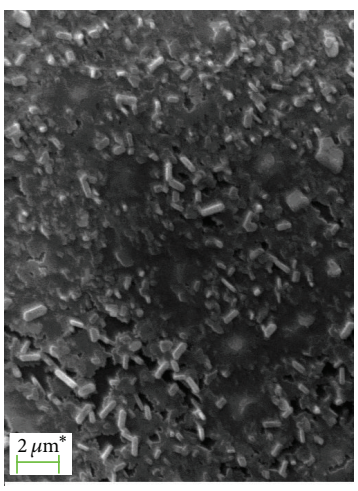

$5 \% \mathrm{ZnO} / \mathrm{PANI}$

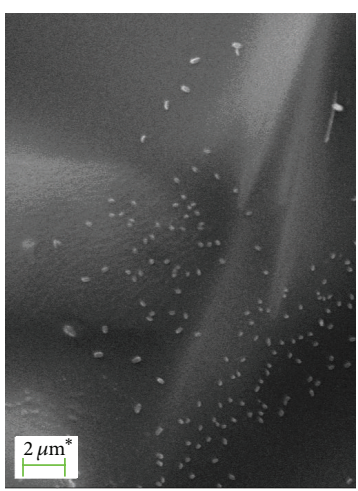

$10 \% \mathrm{ZnO} / \mathrm{PANI}$

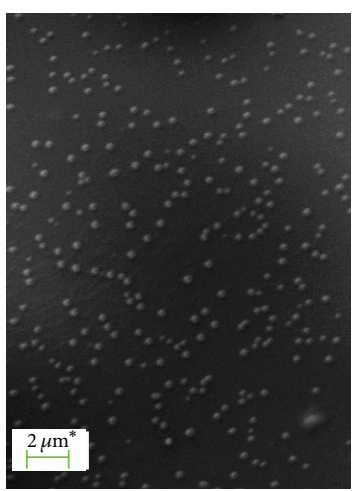

$20 \% \mathrm{ZnO} / \mathrm{PANI}$

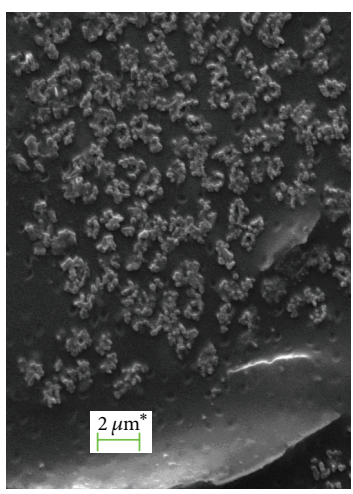

$50 \% \mathrm{ZnO} / \mathrm{PANI}$

Figure 2: SEM image of PANI and PANI/ZnO composites.

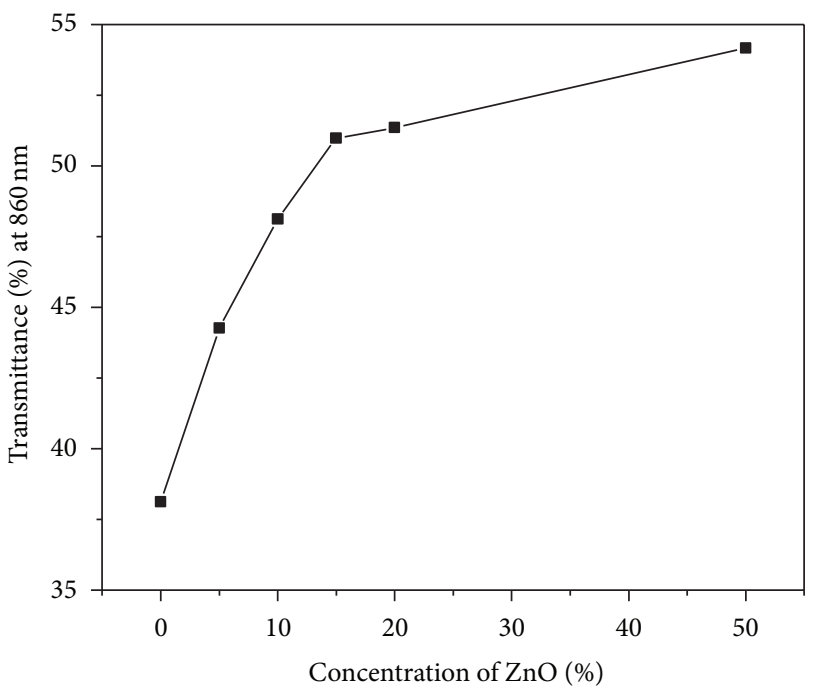

Figure 3: Optical transmittance as a function of $\mathrm{ZnO}$ concentration.

of the curve. The energy gap for pure PANI is observed to be $2.8 \mathrm{eV}$. The higher value of optical band gap is due to the reduction in particle size of the synthesized $\mathrm{ZnO}$ powder. Composites with $5 \mathrm{wt} \%, 10 \mathrm{wt} \%, 15 \mathrm{wt} \%, 20 \mathrm{wt} \%$, and $50 \mathrm{wt} \%$ of $\mathrm{ZnO}$ in PANI exhibit the energy gap of $2.63 \mathrm{eV}, 2.59 \mathrm{eV}$, $2.56 \mathrm{eV}, 2.51 \mathrm{eV}$, and $2.47 \mathrm{eV}$, respectively.

3.3.3. Determination of Refractive Index. Reflectance, transmission, and absorption from any material depend upon the refractive index of that material. Reflectance is given as $R=$ $(n-1)^{2} /(n+1)^{2}$; from this relation, the refractive index as a function of wavelength in terms of reflectance has been derived and reported earlier [14] as $n(\lambda)=-(R+1) \pm$ $\sqrt{\left(-3 R^{2}+10 R-3\right)} / 2(R-1)$. The reflectance of the samples was extracted from the transmittance and absorbance data. Figure 5 shows the variation of refractive index with respect to the concentration of $\mathrm{ZnO}$ at a wavelength of $800 \mathrm{~nm}$. The refractive index is found to be increasing with respect to $\mathrm{ZnO}$ up to $20 \%$ of $\mathrm{ZnO}$ and again decreases at higher dispersion level of $\mathrm{ZnO}$.

3.3.4. Band Gap: Refractive Index Relationship. The refractive index and energy gap of semiconductors represent two fundamental physical aspects that characterize their optical and electronic properties. Optical band gap of a semiconductor determines the threshold for the absorption of photons, and the refractive index determines the transparency of the material. Therefore, the correlation between these two properties obtains the significant importance. Theoretically energy gap-refractive index relationship was obtained by Ravindra et al. [15] using Penn model, $n(Y)=A+B_{1} x+$ $B_{2} x^{2}+B_{3} x^{3}+B_{4} x^{4}$. The same method has been employed to determine the relation between band gap and refractive index theoretically. Figure 6 shows how the refractive index varies with respect to the optical band gap. The relation is well fit into a fourth order binomial equation. The relation between " $x$ " and " $E_{g}$ " is determined as $n=-60.57+94.47 E_{g}-$ $53.99 E_{g}{ }^{2}+13.71 E_{g}{ }^{3}-1.31 E_{g}^{4}$.

3.3.5. Fluorescence Spectroscopic Studies. From previous studies, it is concluded that PANI/ZnO composites exhibit good optical properties and conducting properties. In order to check whether the films can emit radiation, fluorescence studies were carried out. PANI/ZnO nanocomposites were subjected to fluorescence spectroscopy to observe the excitation and emission phenomenon of as-synthesized composites. Figure 7 shows the fluorescence spectra of PANI and PANI/ZnO composites which reveal the emission of radiation in the UV region.

Inset of Figure 7 shows the variation of emission wavelength with respect to $\mathrm{ZnO}$ composition. It is observed that the emission wavelength increases as $\mathrm{ZnO}$ increases up to $20 \%$, and further addition of $\mathrm{ZnO}$ leads to the decrease of emission wavelength. Hence by adding different composition of $\mathrm{ZnO}$ with PANI the radiation of desired wavelength can be obtained. Further it is also observed that the emission is very strong (highly intense) at $20 \%$ of $\mathrm{ZnO}$. Therefore, the PANI 

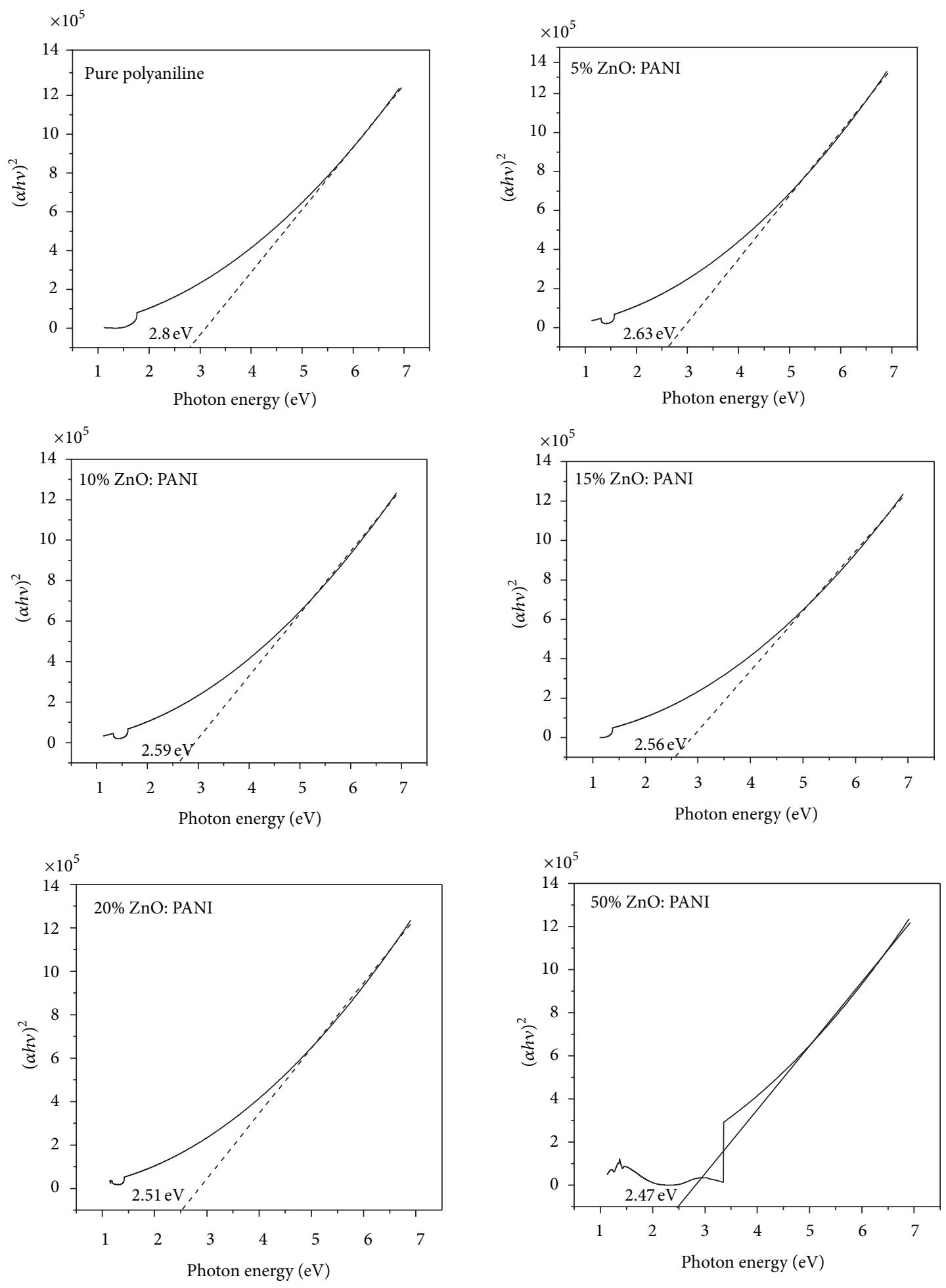

FIgURE 4: Tauc's plot for various compositions of $\mathrm{ZnO}$ in PANI.

film with $20 \%$ of $\mathrm{ZnO}$ emits the radiation of $395 \mathrm{~nm}$ with high intensity.

3.4. Electrical Properties. Figure 8 shows the variation of optical band gap and the electrical conductivity with $\mathrm{ZnO}$ concentration. The band gap is observed to be decreased by the addition of $\mathrm{ZnO}$ nanoparticles. The higher level of incorporation of $\mathrm{ZnO}$ reduces the band gap due to the formation of donor level at the bottom of the conduction band which contributes to electrical conductivity. The widening 


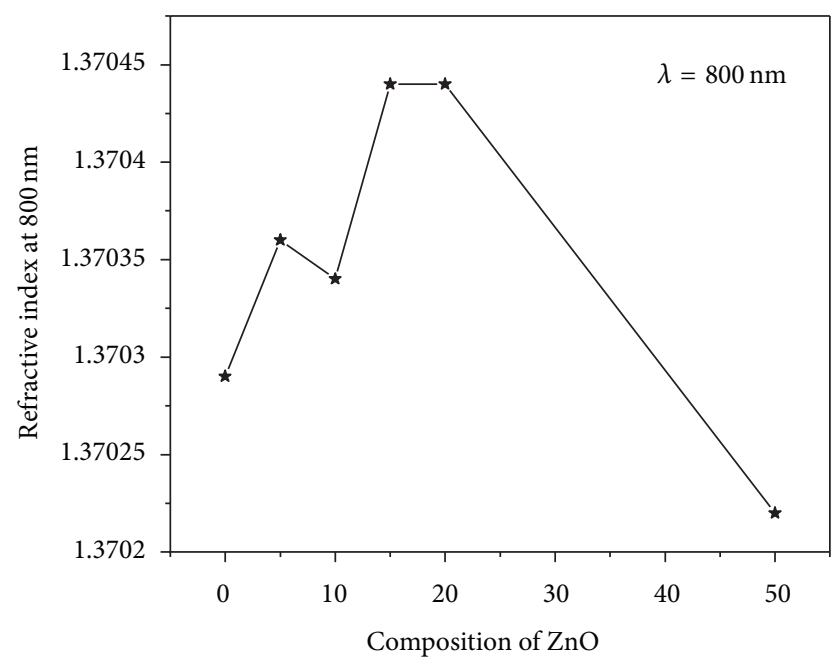

FIGURE 5: Refractive index versus $\mathrm{ZnO}$ concentration at $\lambda=800 \mathrm{~nm}$.

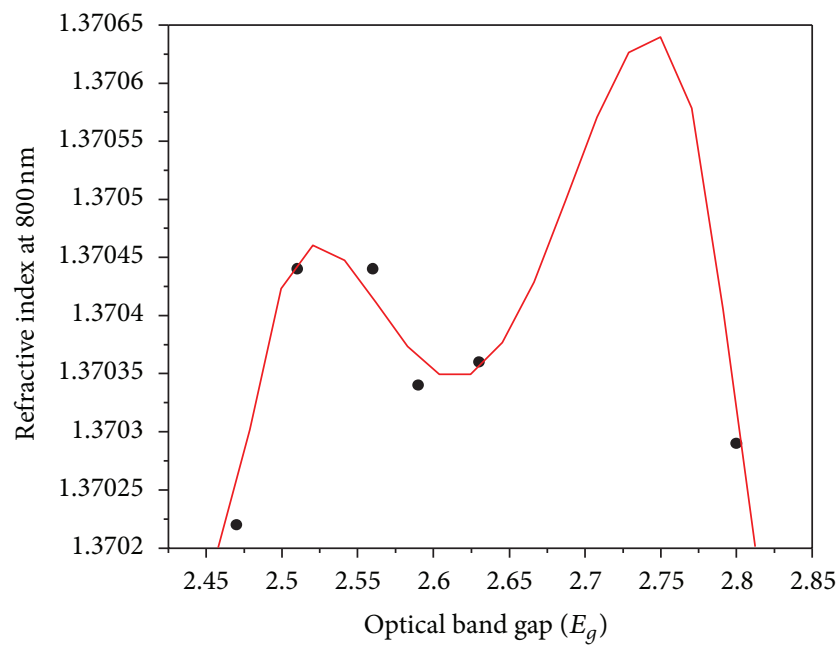

Figure 6: Relation between refractive index and band gap.

of band gap in case of heavily doped semiconductors is due to the blocking of the low-energy transitions by the donor electrons occupying the states at the bottom of the conduction band which is known as the Burstein-Moss effect [16]. According to this effect, the electrical conductivity of the film is expected to be increasing with $\mathrm{ZnO}$ concentration. The electrical conductivity of the film was measured using electrometer. The conductivity was found to be increasing with the increase of $\mathrm{ZnO}$ concentration and well reflects the Burstein-Moss effect.

\section{Conclusion}

Thin films of PANI and $\mathrm{ZnO}$ nanoparticles dispersed PANI were prepared by PVA assisted method on glass substrates. $\mathrm{XRD}$ was carried out to confirm the formation of $\mathrm{ZnO}$ nanoparticles and PANI. The film of PANI and ZnO/PANI nanocomposites was subjected to UV-visible spectroscopy and fluorescence spectroscopy. The dispersion of $\mathrm{ZnO}$
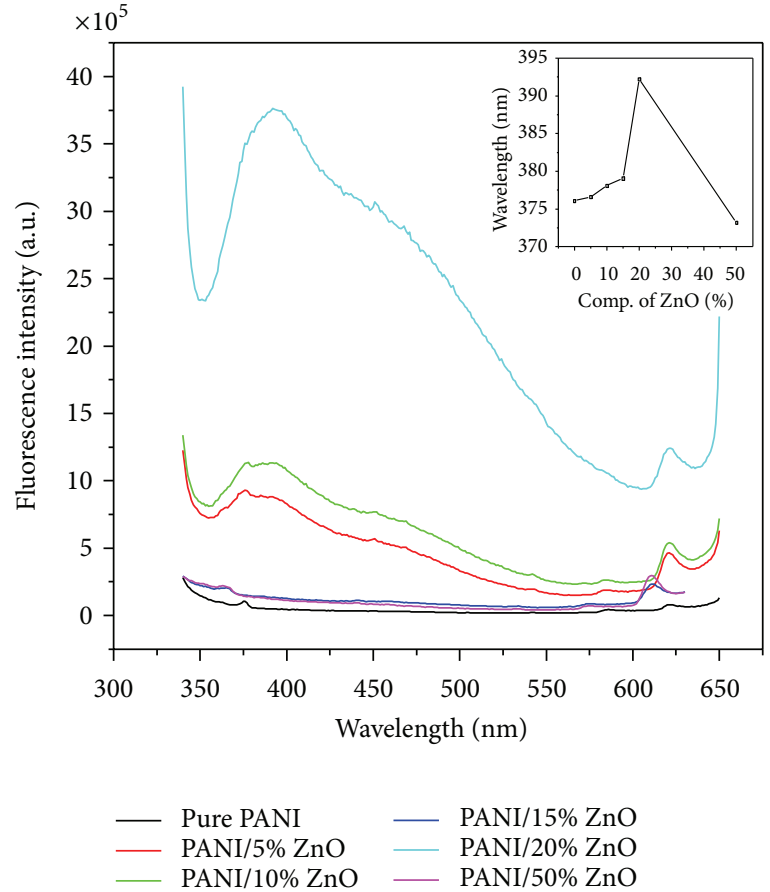

FIGURE 7: Fluorescence spectra of PANI/ZnO films. Inset: Variation of emission wavelength w. r. to $\mathrm{ZnO}$ concentration.

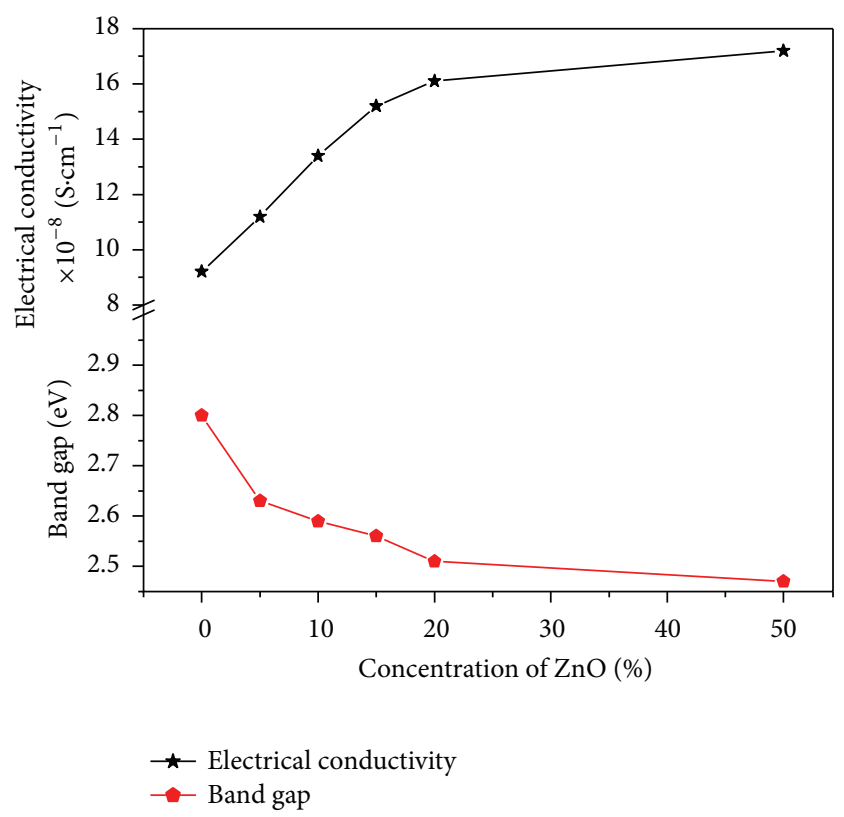

FIGURE 8: Variation of optical band gap and electrical conductivity with respect to $\mathrm{ZnO}$ concentration.

nanoparticles in the PANI matrix improves the transparency and modifies the optical band gap. The electrical conductivity is found to be enhanced by the dispersion of $\mathrm{ZnO}$. Further, the dispersion of $\mathrm{ZnO}$ tunes the emission wavelength and the intensity of the radiation. Hence, it is concluded that 
$\mathrm{ZnO}$ nanoparticle dispersed PANI is a promising material for emissive layer in polymer light-emitting diodes.

\section{References}

[1] A. J. Heeger, "Semiconducting and metallic polymers: the fourth generation of polymeric materials," Angewandte Chemie International Edition, vol. 40, no. 14, p. 2591, 2001.

[2] J. Kido and Y. Iizumi, "Fabrication of highly efficient organic electroluminescent devices," Applied Physics Letters, vol. 73, no. 19, pp. 2721-2723, 1998.

[3] R. H. Friend, R. W. Gymer, A. B. Holmes et al., "Electroluminescence in conjugated polymers," Nature, vol. 397, no. 6715, pp. 121-128, 1999.

[4] H. Hoppe and N. S. Sariciftci, "Organic photovoltaic solar cells: recent advancements in efficiency," Journal of Materials Research, vol. 19, pp. 1924-1945, 2004.

[5] N. Stutzmann, R. H. Friend, and H. Sirringhaus, "Self-aligned, vertical-channel, polymer field-effect transistors," Science, vol. 299, no. 5614, pp. 1881-1884, 2003.

[6] J. H. Burroughes, D. D. C. Bradley, A. R. Brown et al., "Lightemitting diodes based on conjugated polymers," Nature, vol. 347, no. 6293, pp. 539-541, 1990.

[7] C. Winder, A. Andreev, H. Sitter, G. Matt, N. S. Sariciftci, and D. Meissner, "Optoelectronic devices based on para-sexiphenyl films grown by Hot Wall Epitaxy," Synthetic Metals, vol. 139, no. 3, pp. 573-576, 2003.

[8] G. Grem, G. Leditzky, B. Ullrich, and G. Leising, "Blue electroluminescent materials for polymer light-emitting diodes," Advanced Materials, vol. 4, no. 1, pp. 36-37, 1992.

[9] Y. Ohmori, M. Uchida, K. Muro, and K. Yoshino, "Blue electroluminescent diodes utilizing poly(alkylfluorene)," Japanese Journal of Applied Physics, vol. 30, no. 11 B, pp. 19-1941, 1991.

[10] J. Roncali, "Conjugated poly(thiophenes): synthesis, functionalization, and applications," Chemical Reviews, vol. 92, no. 4, pp. 711-738, 1992.

[11] K. S. Cho, J. I. L. Hong, and C. I. Chung, "Effects of $\mathrm{ZnO}$ nano particles on thermal stabilization of polymers," Polymer Engineering and Science, vol. 44, no. 9, pp. 1702-1706, 2004.

[12] A. El-Shaer, A. Dev, J. P. Richters et al., "Hybrid LEDs based on ZnO-nanowire arrays," Physica Status Solidi B, vol. 247, no. 6, pp. 1564-1567, 2012.

[13] S. B. Kondawar, S. D. Bompilwar, V. S. Khati, S. R. Thakrc, V. A. Tabhane, and D. Burghate, "Characterizations of zinc oxide nanoparticles reinforced conducting PANI composite," Archives of Applied Science Research, vol. 2, no. 1, p. 247, 2010.

[14] B. K. Periyasamy, R. S. Jebas, N. Gopalakrishnan, and T. Balasubramanian, "Development of NLO tunable band gap organic devices for optoelectronic applications," Materials Letters, vol. 61, no. 21, pp. 4246-4249, 2007.

[15] N. M. Ravindra, P. Ganapathy, and J. Choi, "Energy gaprefractive index relations in semiconductors-an overview," Infrared Physics and Technology, vol. 50, no. 1, pp. 21-29, 2007.

[16] E. Burstein, "Anomalous optical absorption limit in InSb," Physical Review Letters, vol. 93, no. 3, pp. 632-633, 1954. 

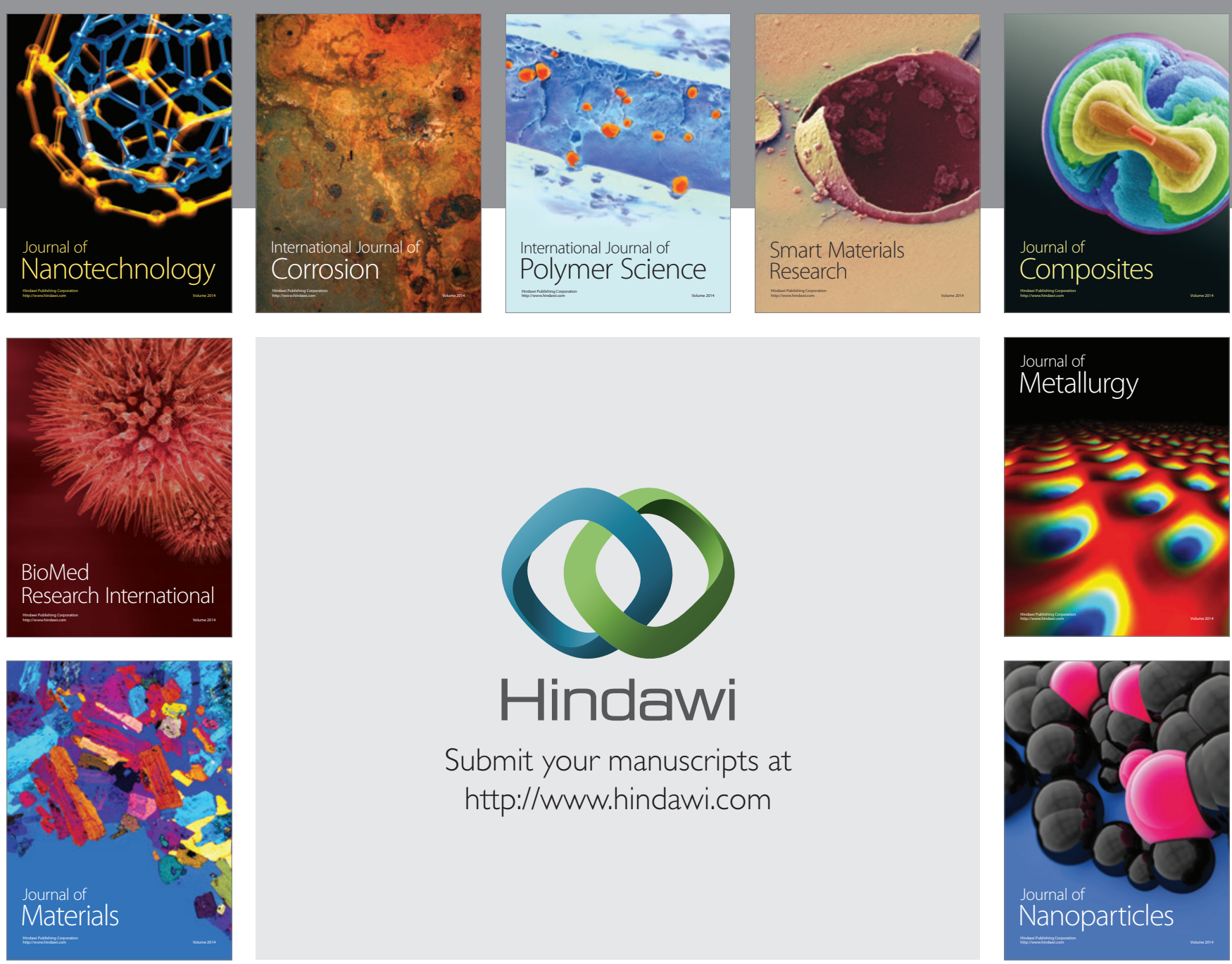

Submit your manuscripts at http://www.hindawi.com
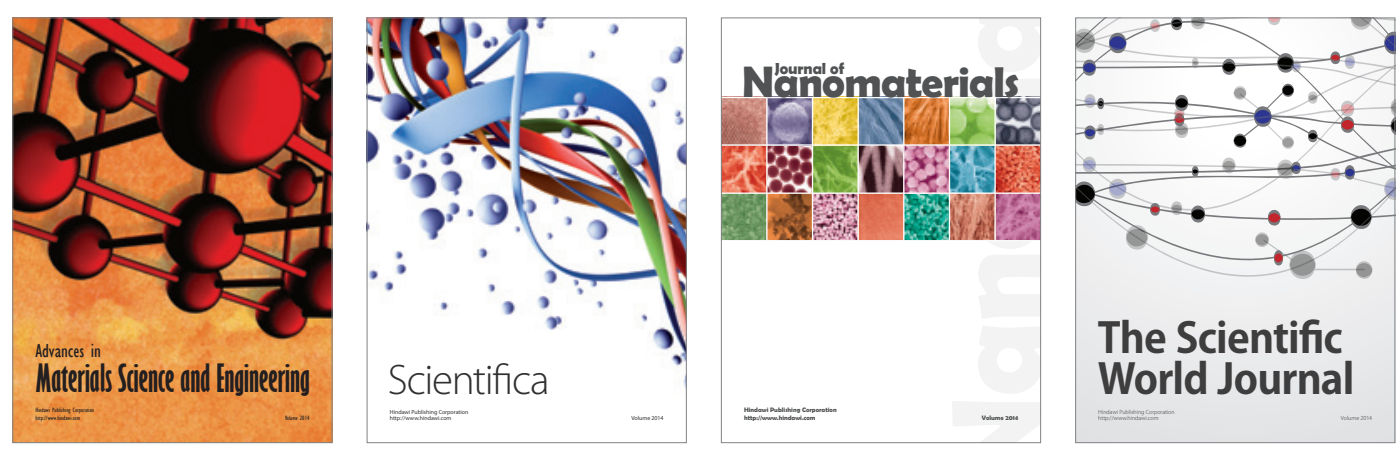

\section{The Scientific World Journal}
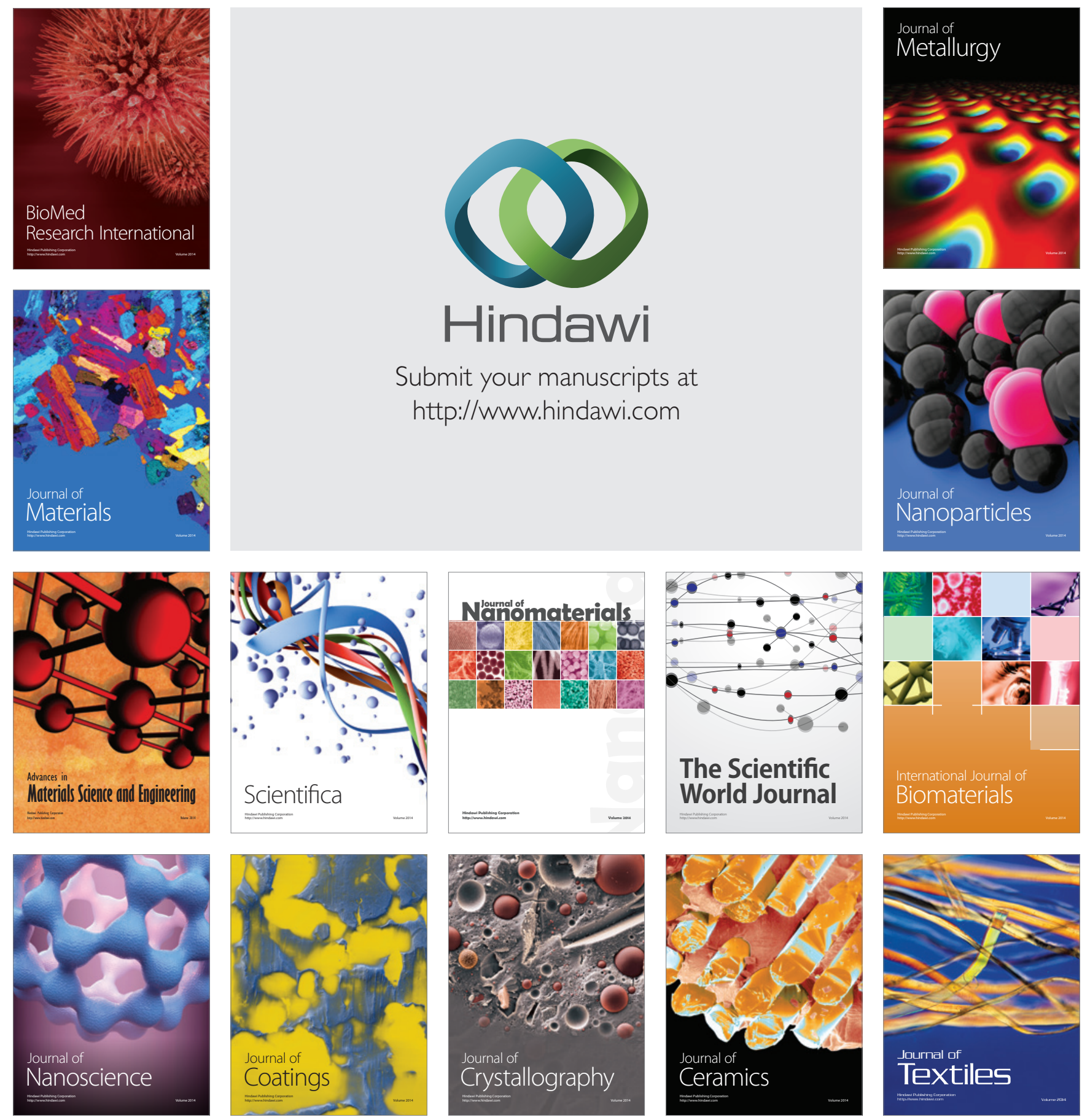